\title{
Low Bit Rate Image Compression using B-Spline Filters and Pre Filtering
}

\author{
P. Ashok Babu \\ Narsimha Reddy Engineering College, \\ Hyderabad, A.P., INDIA.
}

\author{
K.V.S.V.R.Prasad \\ D.M.S.S.V.H.college of Engineering, \\ Machilipatnam, A.P., INDIA
}

\begin{abstract}
In this paper we analyze the performance of B-spline filters, pre filtering and bit-plane coding for low bit rate image compression. The B-spline filters exhibit higher energy concentration in the lower frequency band than other known filters. This property can be exploited for very low bit rate image coding where the higher frequency bands might be very coarsely quantized. The relative information in the low frequency bands of the of the image can be increased by employ of the pre processing technique and bit-plane coding. Averaging technique is employed for image smoothening at low bit rates and bit-plane coding helps removing noise by discarding LSB. In this paper we analyze five techniques for image compression. It is found to be B-spline with one level of decomposition and preprocessing (along with bit-plane coding) gives the appropriate results as compared to the other techniques.
\end{abstract}

Key words: B- Spline filters, filter banks, sub band coding, Pre processing, Image Averaging

\section{INTRODUCTION}

Sub band coding [1] techniques are becoming more popular for compression of speech and video signals. In this technique, an input signal is decomposed into a set of frequency bands by their proper analysis filter banks. In the reconstruction process, these bands are decoded, interpolated, filtered (synthesis) and are added together to represent a replica of the original signal. Several design procedures for the perfect and alias free reconstruction of the filter banks are known [2, 3]. However, higher bands are coarsely quantized for very low bit rate coding applications and then the signal is mainly reconstructed from its lower band. Hence the coding efficiency is improved by using filters that concentrate higher energy in the lower band. Such filters can be of B-Spline type. We have project a two-channel analysis and synthesis filter 3 ). each control vertex influences $\pm \mathrm{k} / 2$ segments of the polygon.

4). B-Spline basis functions always assume non-negative values. banks which are bi orthogonal and use B-Spline FIR filters. In this paper we analyze the error characteristics of these filters and compare their performance for image coding applications against the commonly used filters $[4,5]$.

\section{B-SPLINES}

Polynomials are widely used in the approximation theory and numerical analysis to approximate smooth functions. However, coarse approximation for polynomials of orders greater than 3 or 4 may result in wild oscillations. Fortunately, when the entire domain is segmented, a class of piecewise polynomials can be free of oscillation. BSplines basis functions have such a property and are commonly used to represent curves and surfaces [6]. Let X (u) is the parametric position vector along a curve, as a function of the parameter $u$, and then B-Spline curve is defined by

$$
\begin{gathered}
\mathrm{n}+1 \\
\mathrm{X}(\mathrm{u})=\sum_{\mathrm{i}=1} \quad \mathrm{Y}_{\mathrm{i}} \mathrm{B}_{\mathrm{i}, \mathrm{k}}(\mathrm{u}) \\
\mathrm{u}_{\min } \leq \mathrm{u}<\mathrm{u}_{\max }, \quad 2 \leq \mathrm{k} \leq \mathrm{n}+1
\end{gathered}
$$

Where $\mathrm{Yi}$ are the position vectors of the $\mathrm{n}+1$ polygon vertices and the $\mathrm{B}_{\mathrm{i}, \mathrm{k}}$ are the normalized $\mathrm{B}$-Spline basis functions. The original curve is represented by approximation by a set of data values of polygon vertices. The piecewise polynomial curve is formed by a group of consecutive polynomial segments. The knot vectors are a set of values formed by joints between the segments. The most important properties of the B-Spline basis functions are:

1). The continuity $(C)$ of the surface in each knot vector is two less than the B-Spline orders $(\mathrm{k}), \quad \mathrm{C}^{\mathrm{k}-2}$.

2). The B-Spline order of the curve is restricted to the number of control vertices

5). The curve is invariant with respect to an affine transformation. This implies that any transformation applied to the curve is the same as if it is applied to the control vertices. 


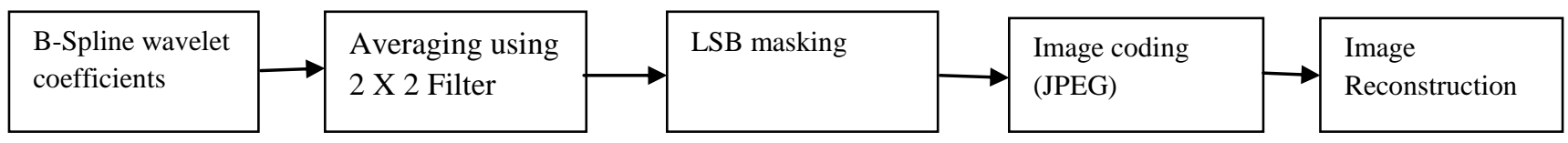

Figure1: Block diagram for the proposed method

\section{PROPOSED ALGORITHM}

We tried compressing the images at low bit rates using 2 levels of spline decomposition and found that at very low bit rates, this can be better as compared to 1 level of decomposition but quality of the image is very poor. High frequency coefficients may not be useful, at low bit rates. Hence input image is smoothed using $2 * 2$ averaging filter and also the LSB of all pixels is masked to 0 as it does not constitute much information. The above obtained image is compressed after 1 level of decomposition and is found to be better. However for 2 levels of decomposition, the quality improved with preprocessing. But it's found to be worse than 1 level of decomposition with preprocessing.

For all the quality factors perform one level of spline decomposition, down sample the image and compress the image using JPEG compressions in both horizontal and vertical directions. Then up sample the image and reconstruct the image assuming high frequency bands as zero. According to proposed algorithm smooth the image using $2 \times 2$ averaging filter in both vertical and horizontal directions. Assume the LSB as zero for all the pixels. Then perform one level of spline decomposition, down sample the image and compress the image using JPEG compressions in both vertical and horizontal directions. Then up sample the image and reconstruct the image assuming high frequency bands as zero.

\subsection{Algorithm without preprocessing}

1).Read the image and initialize spline coefficients.

2).Perform one level of spline decomposition, down sample the image in both vertical and horizontal directions.

3). Compress the image using JPEG compression in both vertical and horizontal directions.

4). Up sample the image in both vertical and horizontal directions.
5). Reconstruct the image assuming high frequency band as zero.

6).Calculate and plot BPP and PSNR.

\subsection{Algorithm with preprocessing:}

1). Read the image and initialize spline coefficients.

2). Smooth the image using $2 * 2$ averaging filter.

3). Make the LSB as zero for all the pixels.

4). Perform 1 level of spline decomposition.

5). Down sample the image.

6). Compress the image using JPEG compressions.

7). Up sample the image

8). Reconstruct the image assuming high frequency band as zero.

9). Calculate and plot BPP and PSNR.

\section{EXPERIMENTAL RESULTS}

In this experiment in combination with a JPEG coder for image compression purposes a B-Spline image approximation scheme is used. The overall compression ratio is the product of $\mathrm{B}$-Spline compression ratio (number of coefficients /number of pixels of the original image) and the compression achieved by the second compressor. In this experiment standard JPEG was used as the B-Spline low frequency band coefficient's image compressor. That is using the B-Spline operator, the dimensions of the image were first reduced from its original size 512 X 512 pixels to 256 X 256 using B-spline filtering. Then perform averaging using 2 X 2 filters and bit-plane coding by removing LSB. The obtained B-Spline coefficients are coded by a JPEG encoder. 
The plots shown below give the results for different standard images comparing all the 5 algorithms

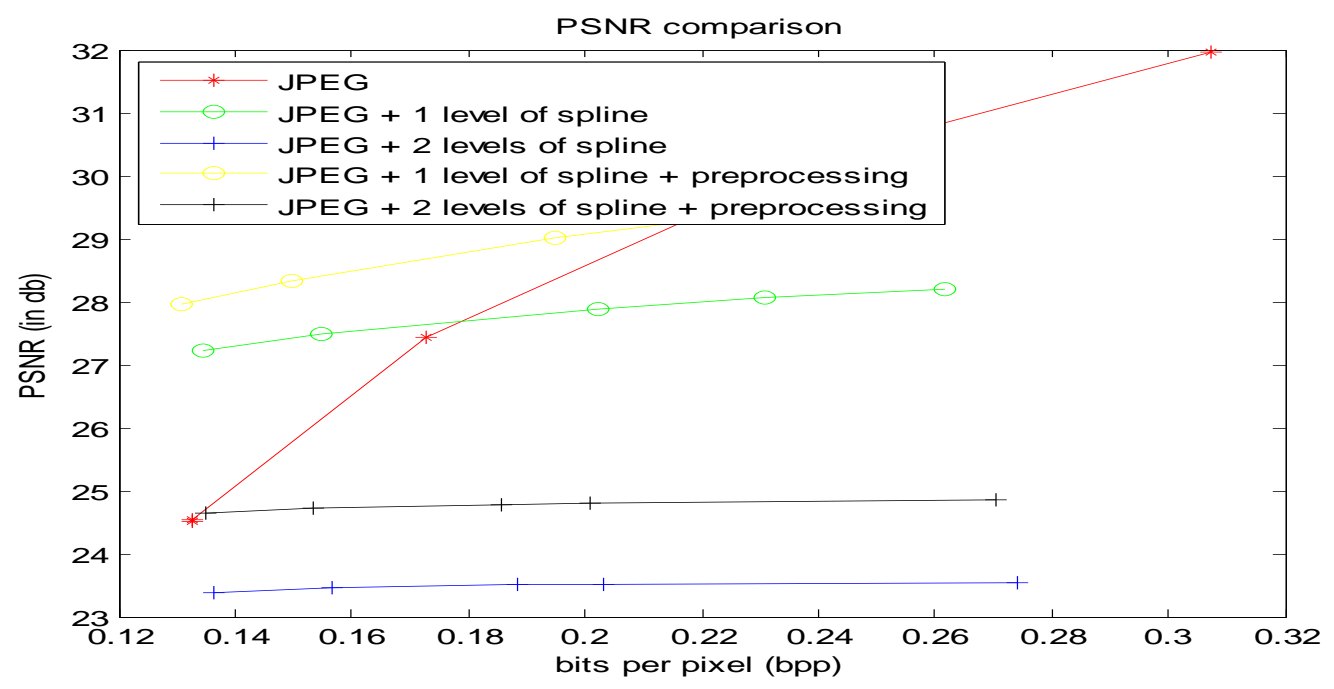

Figure 2: Comparisons of PSNR graphs for Lena with different methods

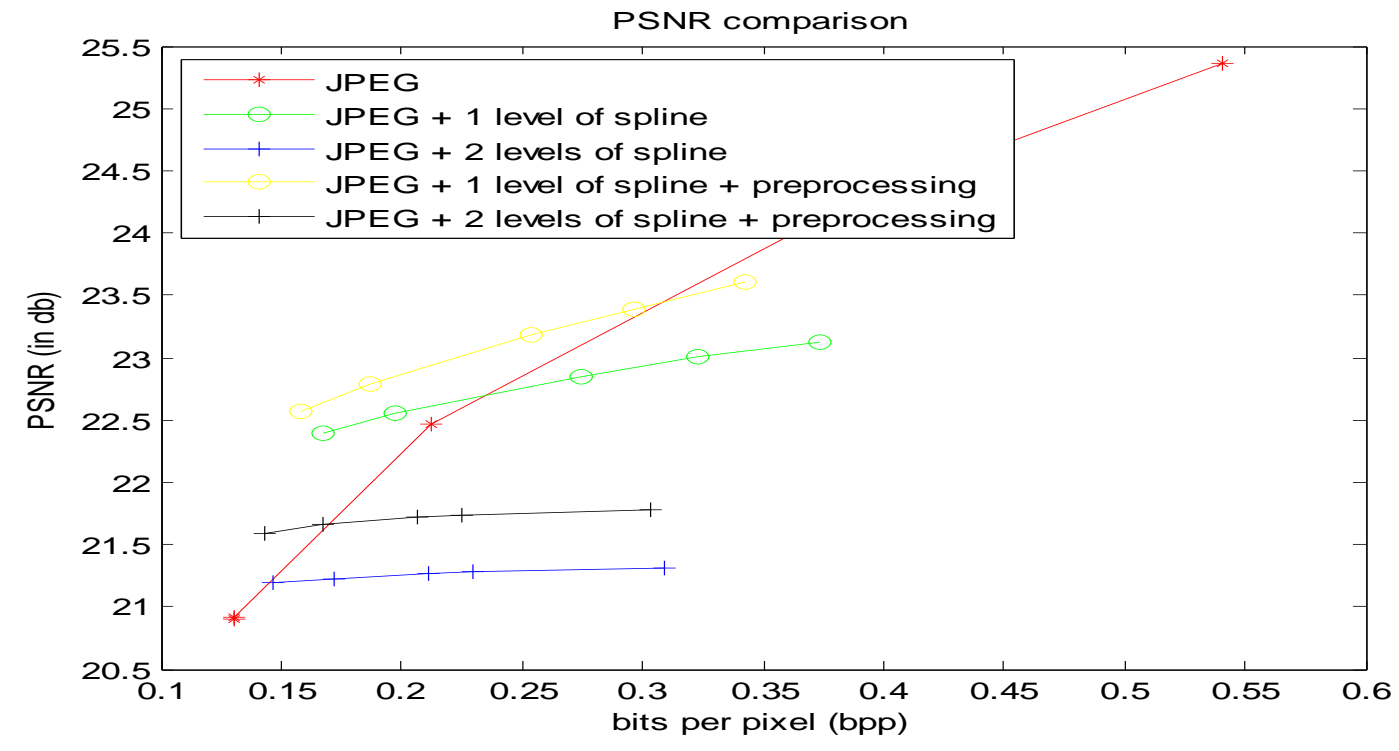

Figure 3: Comparisons of PSNR graphs for Barbara with different methods 


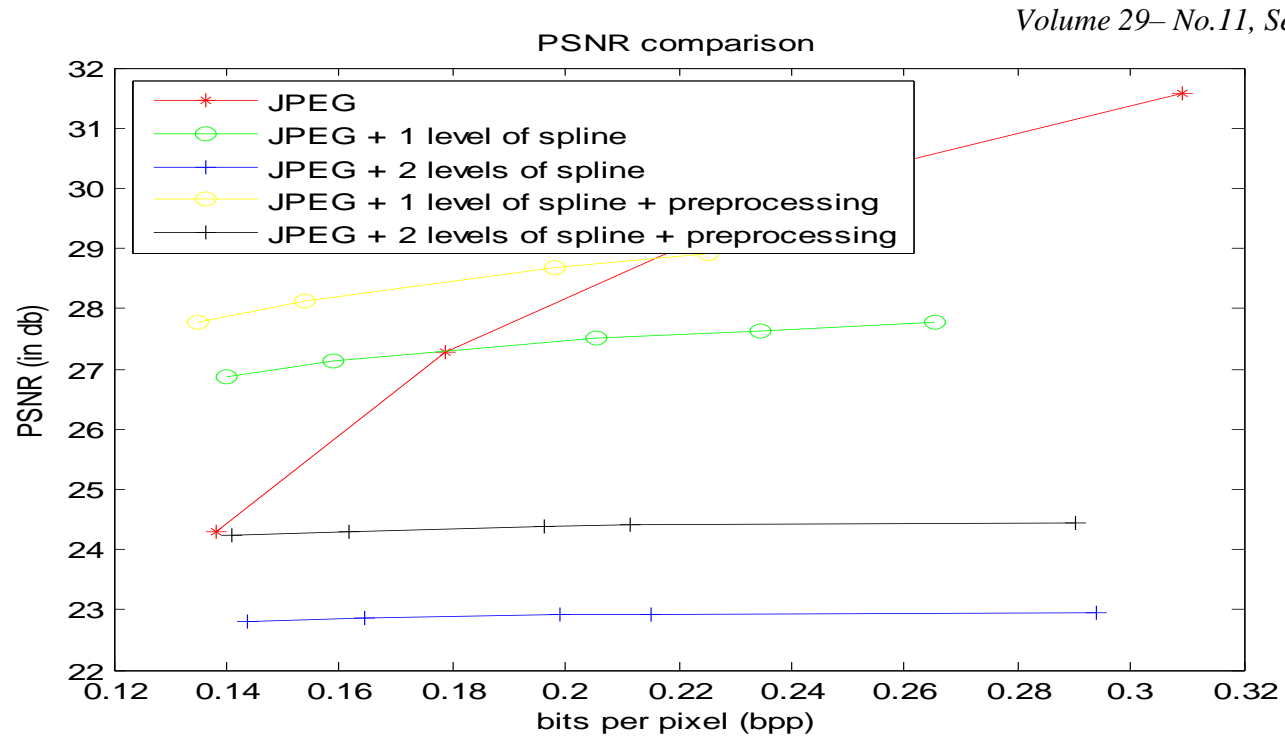

Figure 4: Comparisons of PSNR graphs for peppers with different methods

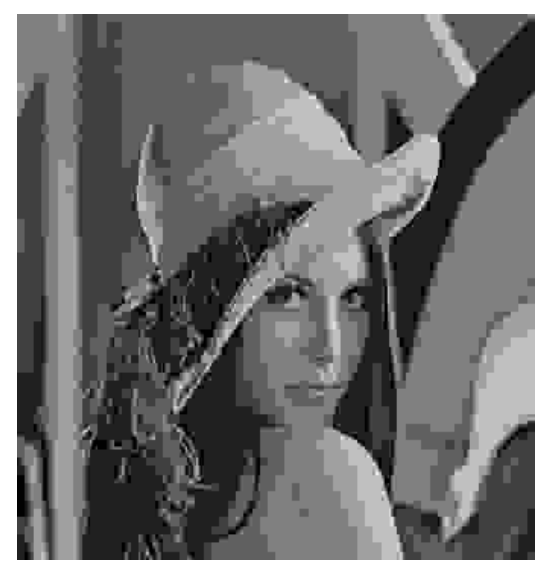

Figure 5: Lena JPEG Coded at 0.14 bpp and PSNR = $25 \mathrm{~dB}$

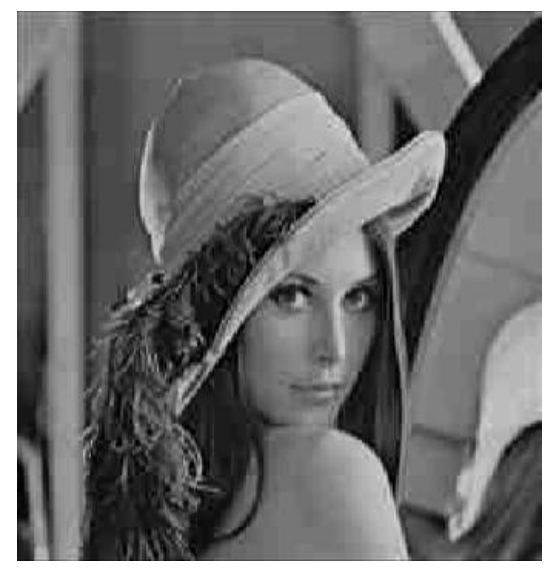

Figure 6: Lena (Spline with 1 level of decomposition + JPEG) coded at 0.14 bpp and PSNR= 26.9 dB 


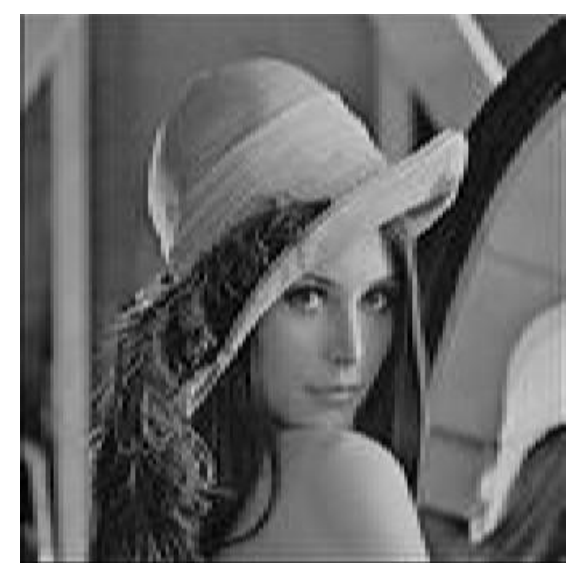

Figure 7: Lena (Spline with 2 level of decomposition + JPEG) coded at 0.14 bpp and PSNR = 23.4 dB

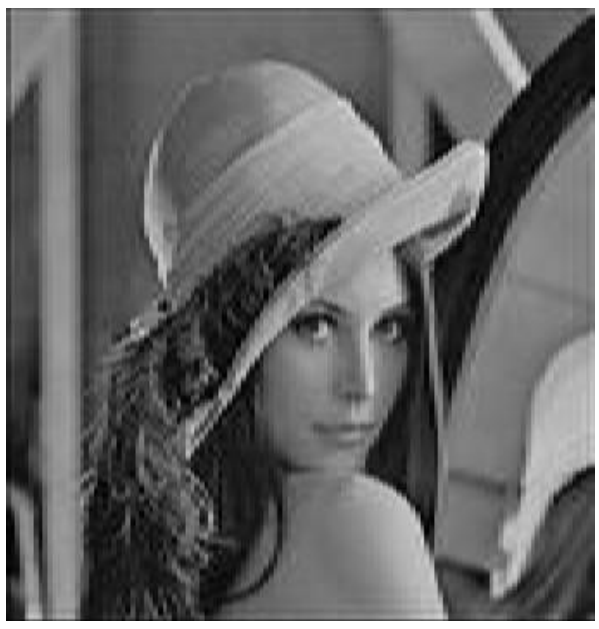

Figure 8: Lena (Spline with 1 level of decomposition + JPEG compression + Preprocessing) coded at 0.14 bpp and PSNR $=28.2 \mathrm{~dB}$

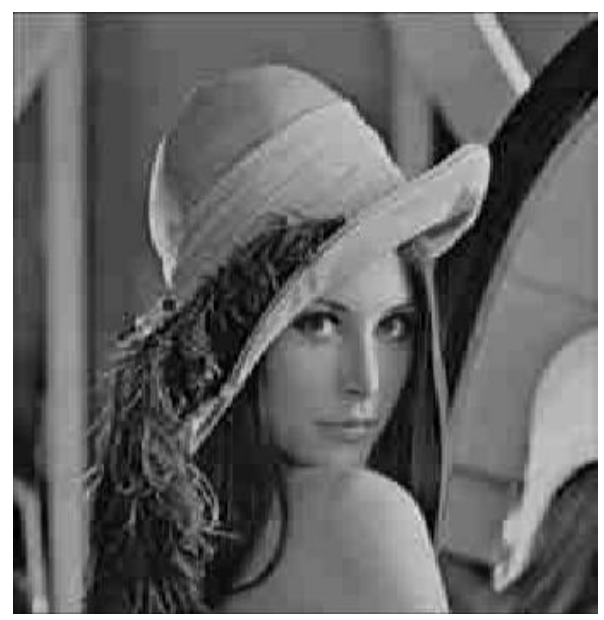

Figure 9: Lena (Spline with 2 level of decomposition + JPEG compression + Preprocessing) coded at 0.14 bpp and PSNR $=24.7 \mathrm{~dB}$ 
The tables shown below give idea about PSNR at low quality factors for different standard images

Table 1: Comparisons of PSNR for Lena with different methods

\begin{tabular}{|c|c|c|}
\hline Name of the Image & Method Used & PSNR(db) at 0.2 bpp \\
\hline Lena & JPEG & 27.9 \\
\hline & JPEG+1 Level of Spline & 27.2 \\
\hline & JPEG + 2 Level of Spline & 25.5 \\
\hline & JPEG+1 Level of Spline+ Pre processing & 28.9 \\
\hline & JPEG+2 Level of Spline + Pre processing & 24.7 \\
& & \\
\hline
\end{tabular}

Table 2: Comparisons of PSNR for Barbara with different methods

\begin{tabular}{|c|c|c|}
\hline Name of the Image & Method Used & PSNR(db) at 0.2 bpp \\
\hline Barbara & JPEG & 22.2 \\
\hline & JPEG+1 Level of Spline & 22.5 \\
\hline & JPEG + 2 Level of Spline & 21.2 \\
\hline & JPEG+1 Level of Spline+ Pre processing & 22.8 \\
\hline & JPEG+2 Level of Spline+ Pre processing & 21.6 \\
\hline
\end{tabular}

Table 3: Comparisons of PSNR for Peppers with different methods

\begin{tabular}{|c|c|c|}
\hline Name of the Image & Method Used & PSNR(db) at 0.2 bpp \\
\hline Peppers & JPEG & 27.8 \\
\hline & JPEG+1 Level of Spline & 27 \\
\hline & JPEG + 2 Level of Spline & 22.9 \\
\hline & JPEG+1 Level of Spline+ Pre processing & 28.6 \\
\hline & JPEG+2 Level of Spline+ Pre processing & 24.2 \\
\hline
\end{tabular}


Fig.2, Fig.3, Fig.4 are the comparisons of PSNR for Lena, Barbara, and peppers. In the comparison graphs we compared five methods. From the graphs we can conclude that at low bits per pixel the proposed method that is JPEG +1 level of spline + preprocessing will gives the better PSNR value.

Fig. 5 is the Lena 512 X 512 image using JPEG coded method at 0.14 bits per pixel and the PSNR value is $25 \mathrm{~dB}$. Fig.6 is the Lena 512 X 512 image using spline with 1 level of decomposition + JPEG method coded at 0.14 bits per pixel and the PSNR value is $26.9 \mathrm{~dB}$. Fig.7 is the $512 \mathrm{X}$ 512 image using spline with 2 level of decomposition + JPEG compression method coded at 0.14 bits per pixel and PSNR value is $23.4 \mathrm{~dB}$. Fig. 8 is the 512 X 512 image using spline with 1 level of decomposition + JPEG compression + pre processing method coded at 0.14 bits per pixel and PSNR value is $28.2 \mathrm{~dB}$. Fig.9 is the 512 X 512 image using spline with 2 levels of decomposition + JPEG compression + pre processing method coded at 0.14 bits per pixel and PSNR value is $24.7 \mathrm{~dB}$.

From the Fig.5, Fig.6, Fig.7, Fig.8, Fig.9, the Fig.8 is more accurate one than the others and the PSNR value is more compared to other methods. So the proposed method that is spline with 1 level of decomposition + JPEG compression + pre processing method will gives the more accurate results than other methods. Table.1, Table.2, Table. 3 are the comparison of PSNR values for Lena, Barbara and Peppers using five methods.

\section{CONCLUSION}

Here Pre processing is nothing but averaging. Hence the high frequency contents are removed by pre processing. Thus we can achieve low bit rates with relatively better quality. By using the two level spline decomposition the quality of the image may degrade. For this reason spline with one level decomposition is found to be the more effective technique than the other methods at low frequencies. Also in bit-plane coding by removing LSB is found to be more efficient due to blind discarding of low information in LSB.

\section{REFERENCES}

[1] R. E. Crochiere and L. R. Rabiner, Multirate Digital Signal Processing. Englewood Cliffs, NJ: Prentice Hall, 1983.

[2] D. Esteban and C. Galand,“Application of quadrature mirror filter banks, m-band extensions and Perfect reconstruction techniques," in Proc. Int. Conf. on ASSP, pp. 191-195, 1977.

[3] P. P. Vaidyanthan, "Quadrature mirror filter banks, M-band extensions and perfect reconstruction Techniques," IEEE ASSP Magazine, pp. 4-20, July 1987.

[4] J. D. Johnston, "A filter family designed for use in quadrature mirror filter banks," in Proceedings of The ICASSP 80 Conference, pp. 291-294, April 1980.

[5] D. Le Gall and A. Tabatabai, "Sub-band coding of digital images using symmetric short kernel filters and arithmetic coding techniques," in Proceedings of the ICASSP 88 Conference, (New York), pp. 761-764, Ap april 1988.

[6] I.J.Schoenberg, "Contributions to the problem of approximation of equidistant data by analytic Functions," Q. Applied Mathematics,vol.4, pp. 4599, 12-141, 1946.

\section{AUTHOR BIOGRAPHIES}

Mr.P.Ashok Babu obtained B.E Degree in 2001 from Andhra University, M.E (Communication Engineering) in 2005 from Osmania University. He is pursuing the Ph.D. from JNTUH university, Hyderabad in Digital Image Processing. He published two papers in International journals and one paper in International Conference. He is member of IACSIT and IAENG . Presently he is working as Assoc. Professor and Head, Department of ECE, Narasimha Reddy Engineering College, Dhullapally, Hyderabad, Andhra Pradesh (state) India.

Dr. K.V.S.V.R Prasad obtained B Sc. Degree in 1963 from Andhra University, B.E (Telecommunication Engineering) in 1967 and M.E (ECE) Microwave Engineering specialization in 1977 from Osmania University. He received the Ph.D. in 1985 from IIT Kharagpur in strip and micro strip transmission lines. He published six papers in IEEE Transactions in MTT, Antenna and Propagation and EMI/EMC and three papers in National Conferences. He is fellow of IETE (life member). He worked in various capacities in the Department of ECE, Osmania University, Hyderabad. Presently he is working as professor and head, Department of ECE, D.M.S.S.V.H. college of engineering, Machilipatnam, Andhra Pradesh (state) India. 